\title{
Blucher
}

\author{
Blucher Proceedings \\ Cuba e Brasil no Século XXI (CBS21) \\ Ciência e Tecnologia na Sociedade do Conhecimento
}

\section{Los Eventos Extremos, Impactos en el Caribe y la Lluvia en la Amazonía}

Dra. Ida Mitrani Arenal ${ }^{1}$, Dr. Oscar Díaz Rodrríguez, Dr. Alejandro Vichot Llamo1, Dr. José Alejandro Rodríguez Zas², Dr. Daniel Martínez Castro1², Dr, Jaci María Vihalva Saraiva ${ }^{4}$

\section{Introducción}

El presente texto aborda un problema actual, que es el posible enlace entre el clima del Caribe, tomando como ejemplo a la isla de Cuba, y el de la Amazonía Brasileña, su evolución futura y los impactos de los eventos extremos en ambas regiones, teniendo como fondo un clima en proceso de rápido cambio.

Estudios realizados por diversos científicos (Curry et al. 2003, Anthes et al. 2006, Mitrani et al., 2008, 2012), muestran la importancia del aporte de agua fresca del río Amazonas a la región del Atlántico Central, de manera tal que la estructura termohalina en la región se altera cuando hay cambios en el caudal del Amazonas. Estas aguas son transportadas por las corrientes ecuatoriales hacia el Caribe (Mitrani et al., 2008), así que los cambios en la estructura termohalina también se manifiestan en las aguas caribeñas.

\footnotetext{
${ }^{1}$ Instituto de Meteorología de la República de Cuba (INSMET), La Habana, Cuba. E-mail: ida.mitrani@insmet.cu. Todos los autores, cuando no se indique expresamente, pertenecen al INSMET.

${ }^{2}$ Empresa CUBAENERGIA, La Habana, Cuba.

${ }^{3}$ Instituto de Meteorología de la República de Cuba (INSMET), La Habana, Cuba. E-mail: daniel.martinez@insmet.cu.

${ }^{4}$ Centro Gestor e Operacional do Sistema de Proteção da Amazônia (CENSIPAM), Manaus, Brasil. E-mail: jmbsarai@yahoo.com.br.
} 
Una disminución en el aporte de agua fresca desde la Amazonía, implica un aumento de la temperatura y la salinidad en las aguas del Atlántico Central y del Caribe, lo cual influye tanto en la circulación termohalina de las aguas como en su estructura vertical (Mitrani et al. 2008) y esto altera de forma significativa el régimen de formación y desarrollo de los ciclones tropicales, así como también sus trayectorias y poder destructivo, lo cual conduce a un aumento de daños provocados por estos eventos, como son las inundaciones costeras en Cuba (Mitrani et al. 2012). Por otra parte, el clima del Caribe debe a su vez influir sobre el de América del Sur, pero existen pocas investigaciones al respecto.

Dada la importancia de los problemas antes expuestos y su gran nivel de incertidumbre, se pretende que científicos de Brasil y Cuba unan sus esfuerzos en el estudio de este interesante enlace entre los climas de ambas regiones y los posible impactos de los eventos extremos que pudieran verse favorecidos por la influencia de los cambios climáticos previstos sobre este enlace.

\section{Regiones de Estudio e Información Utilizada}

En investigaciones realizadas por científicos cubanos, para describir las particularidades de la estructura termohalina en aguas cubanas y sus tendencias temporales, se utilizó la información proveniente de 54 cruceros realizados en aguas cubanas durante el período 1966-2000, con participación de especialistas de diversas instituciones cubanas y de otras naciones como la Unión Soviética y México; a modo de complemento, se utilizaron datos de los archivos de la NOAA, disponibles libremente en su sitio web (NOMAD3, 2013). Se analizaron los cursos anuales de los parámetros que caracterizan a la estructura termohalina y sus posibles cambios temporales, como son: temperatura del agua, espesor de la capa isotérmica, espesor de la capa isopícnica, profundidad de localización de la isoterma de $26^{\circ} \mathrm{C}$, máximo de salinidad y su profundidad de localización Se analizó también la distribución vertical de las masas de agua por las curvas TS. Se abordó la posibilidad de enlace de la estructura termohalina con los cursos anuales de lluvia a escala local y en la Amazonía. También comparó la distribución espacial de los parámetros de la estructura termohalina con la formación y desarrollo de los ciclones tropicales.

Para definir el espesor de la capa uniforme por temperatura y por densidad, se utilizaron los criterios de Levitus (1982) para la elaboración de su "Atlas Climatológico del Océano Mundial"; como frontera inferior de la capa isotérmica, se tomó la profundidad donde la temperatura del agua es menor que la superficial en $0,5^{\circ} \mathrm{C}$ y de la capa isopícnica, donde la densidad convencional es menor en 0,125 con respecto a la superficie. Como frontera inferior convencional de la capa de mezcla, se tomó el estrato más profundo donde la temperatura del agua permanece casi constante durante todo el año, independientemente de las oscilaciones superficiales de los elementos meteorológicos. También se localizó la profundidad 


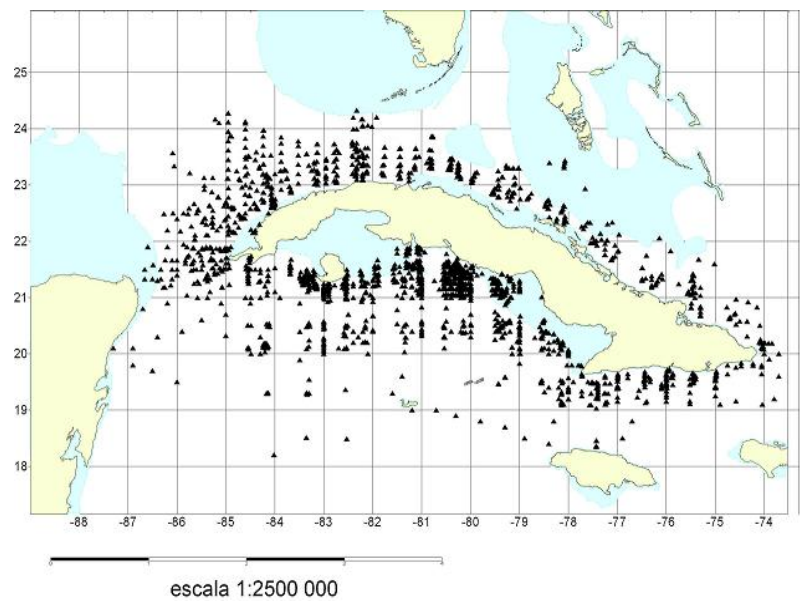

Figura 1: Distribución de las estaciones realizadas durante los 54 cruceros, en el período 1966-2000

de la isoterma de $26^{\circ} \mathrm{C}$, que es la temperatura necesaria para el desarrollo de los ciclones tropicales.

Con la finalidad de entender mejor las particularidades de cada área del entorno cubano, se realizó una división por zonas, atendiendo a la batimetría, forma de la línea costera y presencia de las corrientes permanentes (Figuras abajo).
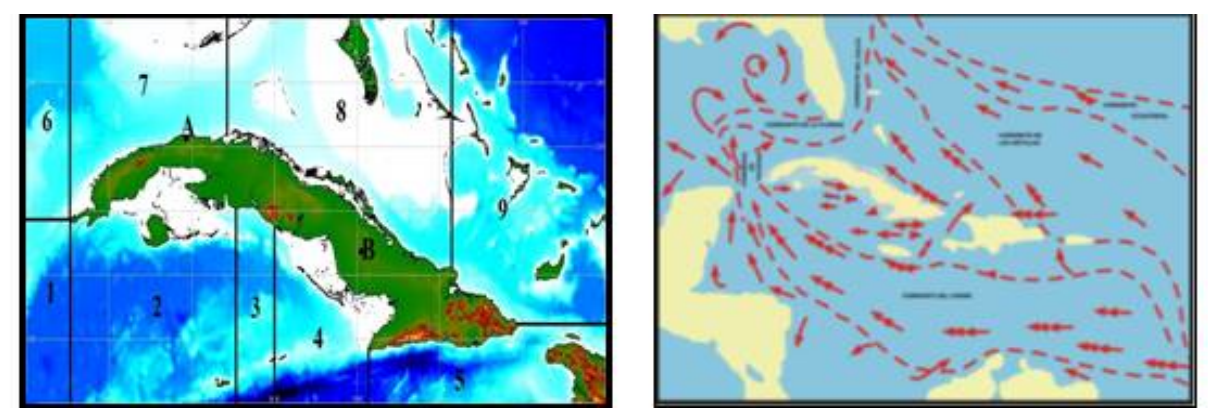

Figura 2: Figura de la izquierda: Batimetría (GEBCO 2003) y zonas de estudio (Mitrani et al. 2008. Figura de la derecha: corrientes permanentes (Mitrani et al. 2008 a). Las cabezas de las saetas indican velocidad de $0.5 \mathrm{~cm} / \mathrm{s}$

Se delimitaron los siguientes tramos:

1. Canal de Yucatán (por el sur), hasta los $87^{\circ} \mathrm{W}$

2. Península de Guanahacabibes - Cayo Ramona.

3. Cayo Ramona - Punta María Aguilar 
4. Punta María Aguilar-Cabo Cruz

5. Cabo Cruz - Punta Maisí y hasta los $73^{\circ} \mathrm{W}$

6. Golfo de México al norte del canal de Yucatán, hasta los $87^{\circ} \mathrm{W}$

7. Península de Guanahacabibes-Punta Hicacos

8. Punta Hicacos - Punta Lucrecia

9. Punta Lucrecia - Punta Maisí y hasta los $73^{\circ} \mathrm{W}$

Para el análisis de las precipitaciones en Cuba, se tomaron dos estaciones meteorológicas con largas series de datos y que se localizan en las áreas centrales de las dos mitades de Cuba, occidental y oriental (Álvarez 2002 a, b) por lo que se garantiza una correcta caracterización de toda la isla. Las estaciones son Casablanca y Canagüey, en las provincias de La Habana y Camaguey respectivamente (letras A y B en la Figura 2).

Para el análisis de la distribución de la precipitación en la Amazonía brasileña, se tomaron los registros de pluviómetros de la red de estaciones (Figura 3) desde 1985 hasta 2000 (Vichot et al. 2011)

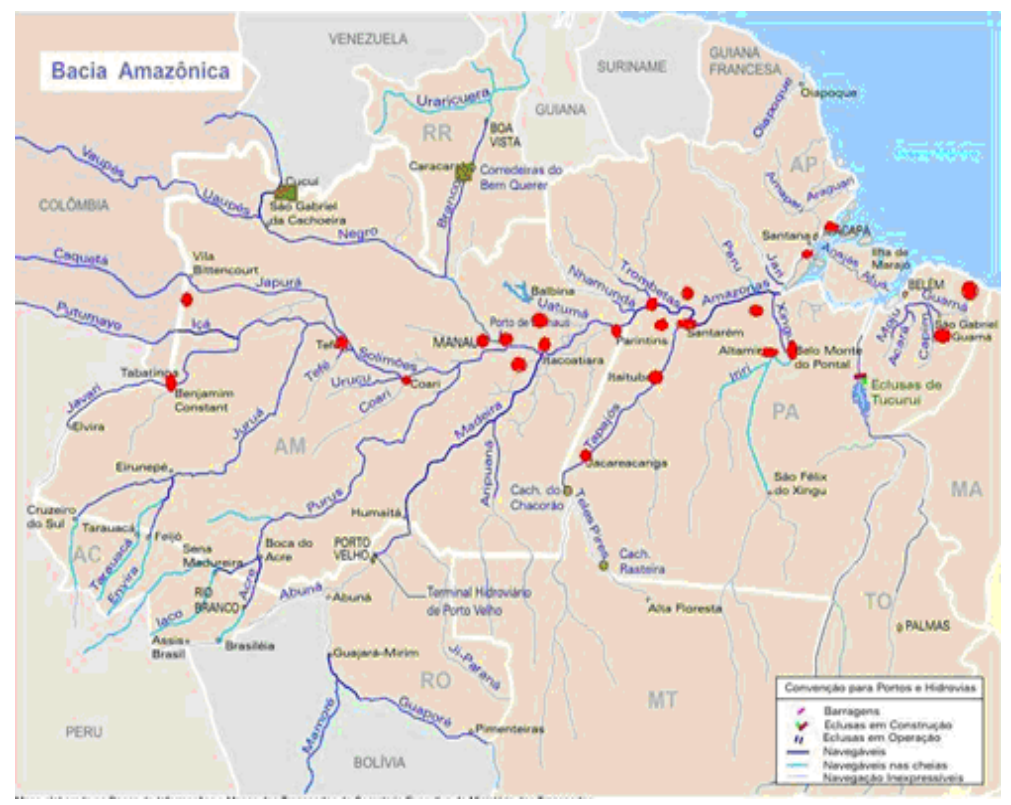

Figura 3: Distribución espacial de las estaciones meteorológicas de la Amazonía, analizadas en el presente texto.

En el presente texto, se mostrarán a modo de ejemplo las estaciones de Altamira y Parintins. 


\section{Resultados y Discusión}

\section{Distribución espacial de los parámetros de la estructura termohalina en aguas cubanas}

En las Figuras 4 y 5 se muestra la distribución espacial de las principales características de la estructura termohalina en aguas cubanas, para las estaciones lluviosa y poco lluviosa, con los datos de los 54 cruceros oceanográficos realizados en 1966-2000 elaborados por Mitrani et al. (2008a) y otros libremente disponibles en Internet, elaborados por Vichot y Mitrani (2011), procesados de conjunto por Rodríguez y Mitrani (2013).
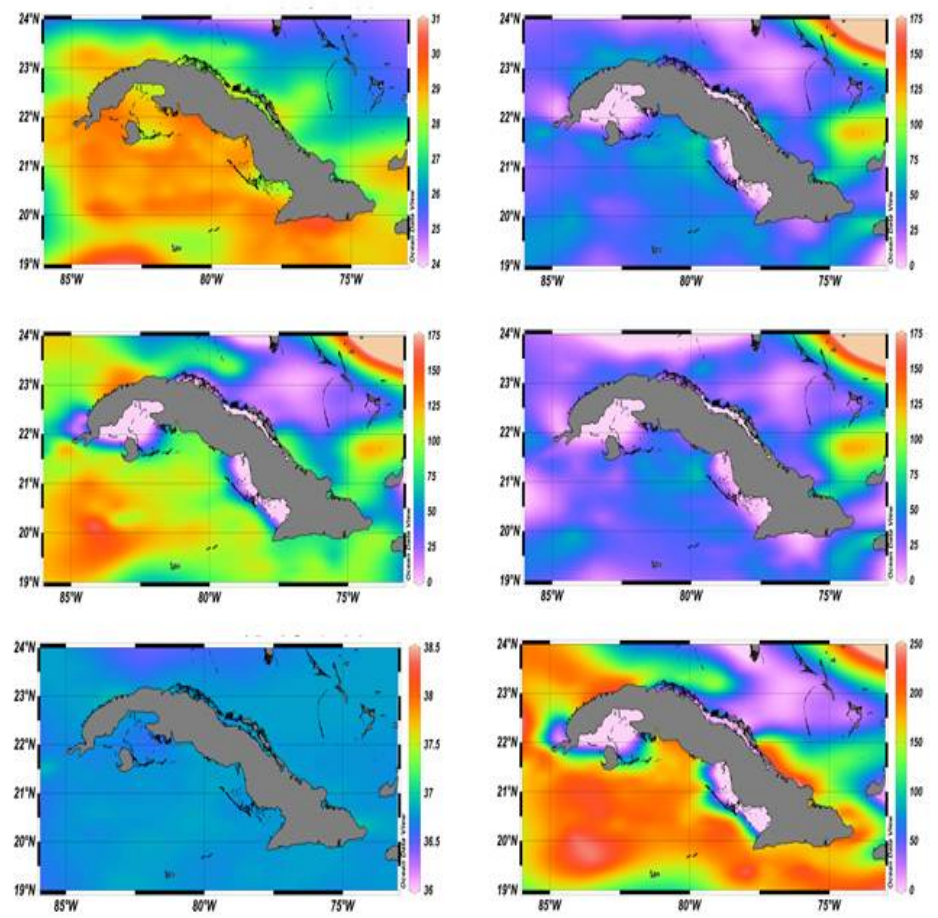

Figura 4: De izquierda a derecha y de arriba a abajo, distribución media del período lluvioso de temperatura superficial, espesor de la capa isotérmica, profundidad de la isoterma de $26^{\circ} \mathrm{C}$, espesor de la capa isopícnica, máximo de salinidad y profundidad de localización del máximo de salinidad.

De estas imágenes, es posible apreciar que la temperatura de la superficie marina como promedio se mantiene entre $26^{0}$ y $30^{\circ} \mathrm{C}$ prácticamente sobre todas las aguas aledañas a Cuba. Los espesores de la capa isotérmica, de la capa isopícnica (capa de densidad constante) y de la profundidad de la isoterma de $26^{0} \mathrm{C}$, como 

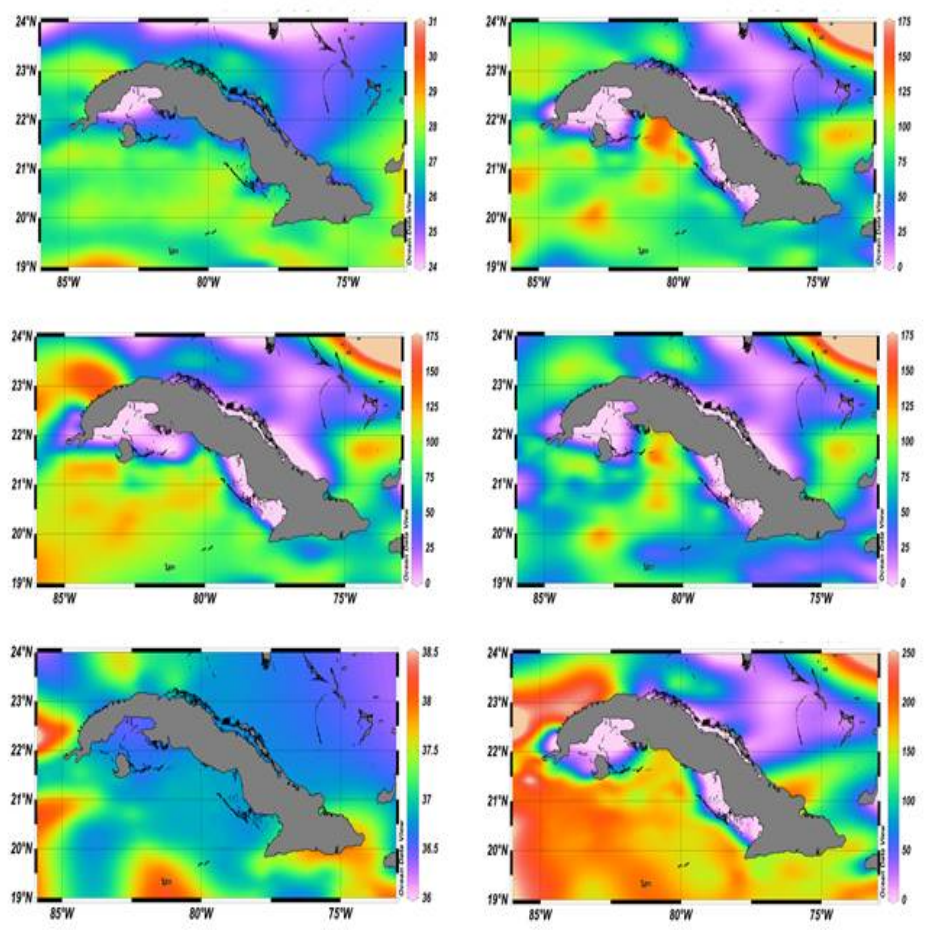

Figura 5: De izquierda a derecha y de arriba a abajo, distribución media del periodo poco lluvioso de temperatura superficial, espesor de la capa isotérmica, profundidad de la isoterma de $26^{\circ} \mathrm{C}$, espesor de la capa isopícnica, máximo de salinidad y profundidad de localización del máximo de salinidad.

promedio son del orden de varias decenas de metros. El máximo de salinidad presenta valores altos, entre 36 y 38 psu, y su profundidad de localización, en general es mayor de los $200 \mathrm{~m}$. Para todos estos parámetros, aunque sus valores son altos en toda el área de las aguas que rodean al Archipiélago Cubano, los máximos se localizan al sur y al norte de las provincias occidentales cubanas, en coincidencia con la región más afectada por los ciclones tropicales.

\section{Referencias}

La información citada en este trabajo se puede encontrar en las publicaciones de los autores.

Recife, Olinda, Maceió, 2014 